\title{
Effects of Gradual and Rapid Variable-Interval Schedule Thinning on Concurrent Schedule Performance in Pigeons
}

\author{
Stephanie L. Kincaid
}

Follow this and additional works at: https://researchrepository.wvu.edu/etd

\section{Recommended Citation}

Kincaid, Stephanie L., "Effects of Gradual and Rapid Variable-Interval Schedule Thinning on Concurrent Schedule Performance in Pigeons" (2013). Graduate Theses, Dissertations, and Problem Reports. 7323. https://researchrepository.wvu.edu/etd/7323

This Thesis is protected by copyright and/or related rights. It has been brought to you by the The Research Repository @ WVU with permission from the rights-holder(s). You are free to use this Thesis in any way that is permitted by the copyright and related rights legislation that applies to your use. For other uses you must obtain permission from the rights-holder(s) directly, unless additional rights are indicated by a Creative Commons license in the record and/ or on the work itself. This Thesis has been accepted for inclusion in WVU Graduate Theses, Dissertations, and Problem Reports collection by an authorized administrator of The Research Repository @ WVU. For more information, please contact researchrepository@mail.wvu.edu. 


\title{
Effects of Gradual and Rapid Variable-Interval Schedule Thinning on Concurrent Schedule Performance in Pigeons
}

\author{
Stephanie L. Kincaid \\ Thesis submitted to the \\ Eberley College of Arts and Sciences \\ at West Virginia University \\ in partial fulfillment of the requirements \\ for the degree of
Master of Science
in
Psychology

Kennon A. Lattal, Ph.D., Chair Claire St. Peter, Ph.D. Amy Fiske, Ph.D.

Department of Psychology

Morgantown, West Virginia

2013 


\begin{abstract}
All rights reserved
INFORMATION TO ALL USERS

The quality of this reproduction is dependent upon the quality of the copy submitted.

In the unlikely event that the author did not send a complete manuscript and there are missing pages, these will be noted. Also, if material had to be removed, a note will indicate the deletion.
\end{abstract}

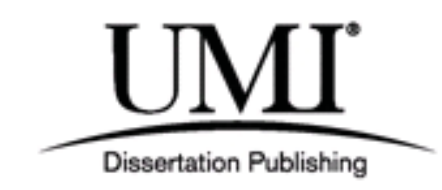

UMI 1549757

Published by ProQuest LLC (2013). Copyright in the Dissertation held by the Author.

Microform Edition (c) ProQuest LLC.

All rights reserved. This work is protected against unauthorized copying under Title 17, United States Code

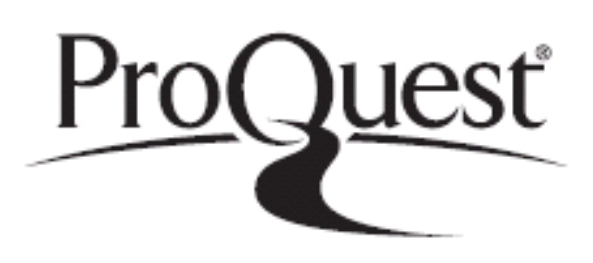

ProQuest LLC.

789 East Eisenhower Parkway

P.O. Box 1346

Ann Arbor, MI 48106 - 1346 


\author{
Abstract \\ Effects of Rapid and Gradual Variable-Interval Schedule Thinning \\ on Concurrent Schedule Performance in Pigeons \\ Stephanie L. Kincaid
}

Schedule thinning procedures are usually implemented with the goal of maintaining behavior despite decreasing reinforcement. The effectiveness of these procedures appears to depend largely at the rate at which reinforcement is decreased, with thinning procedures that decrease reinforcement too rapidly resulting in response elimination. Despite the importance of schedule thinning rate in determining the efficacy of these procedures, different rates of thinning are seldom compared directly in terms of their effects on the elimination of the response. The present study examined the effects of different rates of thinning on response elimination in the context of alternative reinforcement. Pigeons responded on two concurrent variable interval schedules in a Findley (1958) arrangement. One component schedule was thinned at across sessions, while the other component schedule remained constant. More gradual thinning resulted in more gradual response elimination; however, this effect could be explained entirely by the persistence of reinforcement in more gradual thinning conditions.

Keywords: schedule thinning, concurrent schedules, behavioral contrast, extinction 


\section{Table of Contents}

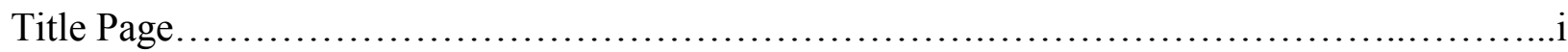

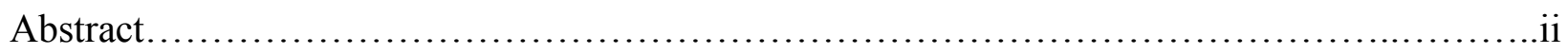

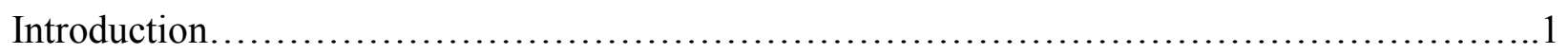

Literature Review...................................................................

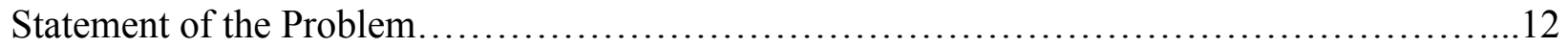

Method......................................................................... 15

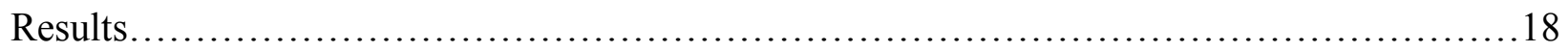

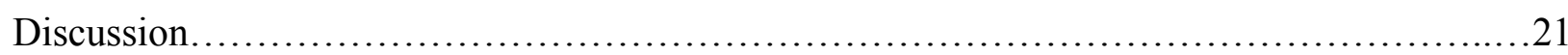

References.................................................................. 34

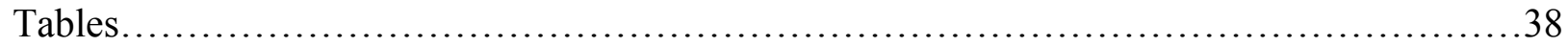

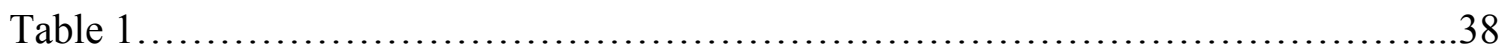

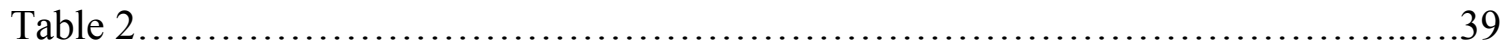

Figures..................................................................... 40

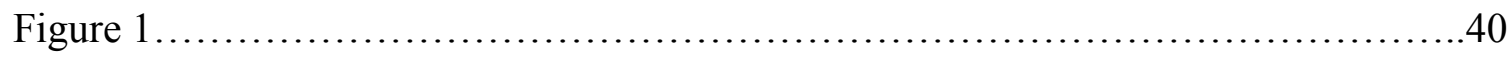

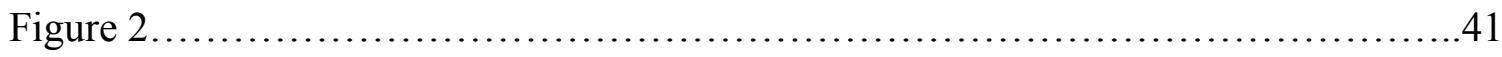

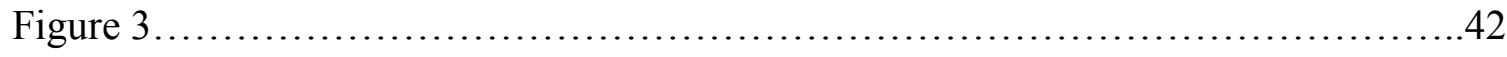

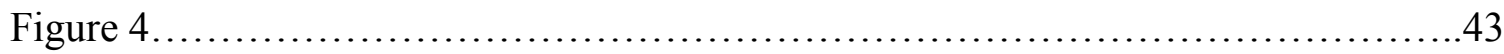

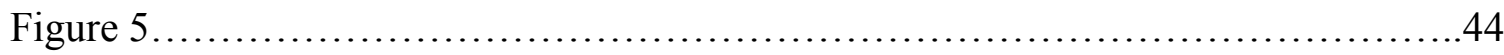

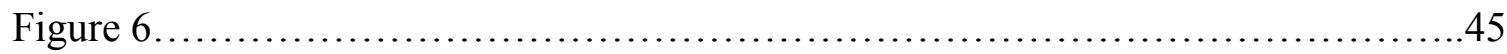

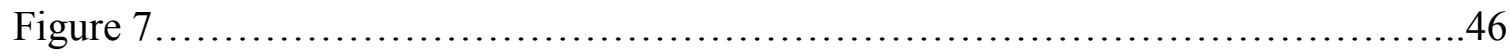




\section{Effects of Gradual and Rapid Variable-Interval Schedule Thinning}

On Concurrent Schedule Performance in Pigeons

The transition from conditions in which reinforcement is presented at a high rate (relatively "rich" schedules) to conditions in which reinforcement is a presented at a low rate ("lean" schedules) is a common one, in the lab and life. Dieting, decreasing addictive drug use, and graduating from undergraduate to graduate school all involve transitioning between different schedule conditions. These transitions may involve decreasing reinforcement rates for one behavior, while reinforcement rates remain constant or even increase for other behavior. Rich to lean schedule condition transitions are observed in treatment settings and in the basic research lab. In clinical settings, treatment often involves rich schedules of reinforcement that may require substantial resources to implement and then sustain. As a result, the original treatment package may be difficult to maintain over long periods of time. In this case, the transition between a treatment and maintenance program may involve a rich to lean schedule transition. In the basic research laboratory, pretraining for an experiment often involves the initial presentation of reinforcement on a rich schedule that is subsequently decreased to a leaner schedule parameter that maintains behavior on a baseline for study. In all of these cases, the goal is to maintain behavior in the face of decreasing reinforcement.

The apparently successful method for achieving this goal is to create a smooth transition between rich and lean schedule conditions that involves gradually decreasing the frequency of programmed reinforcement presentations per unit time. This technique is termed schedule "leaning," "thinning," or "fading." Controlling the rate of the transition between the initial (rich) schedule and the terminal (lean) schedule is an essential feature of these techniques. If the transition is too rapid, behavior is disrupted. This disruption may take the form of in an increase 
in other previously or currently reinforced (and possibly undesirable) behavior as seen in many examples of schedule thinning in the applied literature, or extinction of the target response as observed in ratio strain. Alternately, if the transition is too gradual, resources may be wasted. In both basic and applied research settings, often the goal is to make the transition as short as possible. Basic researchers, for example, may attempt to proceed quickly through the pretraining phase of an experiment in order to conserve resources and begin collecting data related to the primary phenomena of interest as soon as possible. In clinical settings, shorter transition to a maintenance program can save resources required for treatment administration, which can be beneficial for both the clinician (e.g. in terms of minimizing treatment cost) and the patient (e.g. by decreasing the amount of time that must be allocated for treatment).

Perhaps as a result of this goal, schedule thinning procedures often are marginalized. Schedule thinning is viewed as a necessary bother that must be dealt with along the way to baseline performance or long-term treatment success. In this way, schedule thinning procedures are treated much like other transition states in behavior analysis: as a nuisance between periods of steady state behavior, and only infrequently studied as primary subject matter in their own right. Consequently schedule thinning procedures, though apparently effective, are based on little basic research. Schedule thinning often is done, but little understood.

That schedule thinning procedures have been relegated to this status has resulted in a lack of information that may be helpful on both conceptual and pragmatic levels. The search for understanding the nature of behavior change under schedule thinning conditions raises questions about the mechanics of schedule transitions which have only begun to be addressed by behavior scientists. Additionally, understanding schedule thinning procedures could aid the development of more effective methods of their implementation in treatment settings. In this review, several 
examples of schedule thinning procedures are considered as they have been used in basic and applied research settings, as well as several other examples of gradual and abrupt behavioral transitions. There is a particular focus on gaps in the literature of these areas that invite further research. Finally, the results of an experiment that evaluated rates of schedule thinning in the context of alternative reinforcement are reported.

\section{Literature Review}

This review considers schedule thinning and other related examples of behavioral transitions. First, the effects of extinction are considered as an example of rapid schedule thinning. Then, examples of gradual transitions from research concerning punishment, errorless discrimination learning, and shaping are discussed. Schedule thinning procedures as they are used in basic and applied research settings then are considered. Finally, techniques for controlling the rate of schedule transitions are discussed.

\section{Extinction}

To understand the effects of transitioning from rich to lean schedule conditions as they may be observed under schedule thinning conditions, a logical starting place is with the transition to extinction. Extinction is essentially "extreme schedule thinning" in which the procedural transition between rich and lean conditions is rapid (immediate) and the lean schedule is extremely lean (nonexistent). Under extinction, responding is disrupted. This disruption may take several nonmutually exclusive forms, including a decline in the rate of the target response undergoing extinction, resurgence, and behavioral contrast.

The primary effect of removing reinforcement is extinction of the response, or the decline of responding to low or nonexistent levels. Several variables influence the rate of the decline. If a response takes longer to decline, or "extinguish," we say the response is "resistant to 
extinction." Behavior maintained by schedules of intermittent reinforcement is more resistant to extinction than that maintained by reinforcing each response (so-called continuous reinforcement or fixed-ratio 1 schedules). This phenomenon is known as the partial reinforcement effect (e.g., Nevin 1988). Additionally, behavior previously maintained by schedules arranging more frequent, greater magnitude. And more immediate reinforcement is more resistant to extinction than behavior previously maintained by schedules arranging less frequent, smaller magnitude, and more delayed reinforcement (Nevin, 1974).

Extinction also may result in the recurrence of previously reinforced behavior, a phenomenon labeled resurgence. Resurgence is defined as the return of a previously reinforced (but currently extinguished) response when a more recently reinforced response is extinguished (Lattal \& St. Peter Pipkin, 2009). Resurgence typically is observed in a three-stage procedure. In the first, a response is reinforced. In the second, a topographically different response is reinforced and the original response is no longer reinforced. In the third and final, extinction is in effect for both responses. Recurrence of the original response during the third phase is labeled as resurgence.

Additionally, extinction can result in an increase in other behavior that is currently being reinforced in a different context, a process described as behavioral contrast. Behavioral contrast is an interactional effect in which response rate during the presentation of one stimulus changes in the opposite direction of responding generated by changes in the schedule associated with another stimulus (Reynolds, 1961). For example, when reinforcement is no longer presented in the presence of one stimulus, response rate in the presence of another stimulus increases above the rate maintained when responding was reinforced in the presence of both of the stimuli (Reynolds, 1961). 
Response elimination, resurgence, and behavioral contrast all are outcomes of the removal of reinforcement. Reinforcement, however, does not need to be stopped completely for these extinction-like effects to appear. Disruption of responding also has been observed in transitory schedule conditions, in which reductions in reinforcement rate or stimulus changes result in extinction-like effects. If the goal is to maintain behavior across transitory conditions, historically the approach has been to change the environmental conditions, but change them gradually. Some empirical examples of this approach are considered next.

\section{Gradual Transitions}

One example of a case in which responding is maintained under gradually changing stimulus conditions involves gradual changes in punishment intensity. Miller (1960) found that responding could be maintained in the face of response-dependent shock presentations if the shock intensity was increased gradually. Miller trained rats to run an alley in which reaching the goal box was reinforced with food. For one group, reaching the goal box also resulted in shock presentation, with the intensity of the shocks increased gradually across several days. During test trials, reaching the goal box resulted in shock presentation of the same intensity (400 volts) for all subjects. Miller found that rats that had a history of gradually increasing shock intensity maintained higher run speeds in the test trials than rats that were abruptly exposed to the terminal shock intensity. These results were later corroborated by Karsh (1963).

Errorless discrimination learning is another example in which gradually changing stimulus conditions can shape up target responding. Terrace (1963) presented pigeons with stimuli that initially differed in terms of wavelength. Responding in the presence of one stimulus was reinforced $(\mathrm{S}+)$ and responding in the presence of the other stimulus was not reinforced (S-). Terrace found that if the stimuli initially differed in brightness as well as wavelength (S+ 
brighter than S-), but the brightness of the stimuli gradually increased across sessions, the pigeons made fewer errors (responses in the presence of S-) than pigeons that were exposed initially to stimuli of different wavelength but the same brightness. The gradual introduction of the S- in this procedure resulted differential responding that was obtained with very few errors.

The shaping of behavior through the differential reinforcement of successive approximations to the target response is a protoypical example of the effectiveness of gradual transitions. Galbicka (1994) noted that one of the "golden rules" of shaping is to gradually increase the response criterion by using small steps between successive approximations. Although relatively large shifts in the response criterion may result in effective shaping (Eckerman, Heinz, Stern, \& Kowlowicz, 1980), step sizes that are too large may drastically decrease the probability of a criterional response, and therefore the probability of reinforcement. The transition between step sizes must be gradual enough for responding to contact the reinforcement contingency. But how gradual is gradual enough? Ultimately, the speed of the shaping transition is a balance that is titrated by the interaction between the shaper and the behavior being shaped.

The challenge of maintaining this balance has prompted some to refer to shaping as more of an art form than a science (Galbicka, 1994). One attempt to formalize the shaping process has been developed in percentile schedules. In a percentile schedule, the experimenter specifies the percentile at which a particular range of responding (usually, a range of inter-response times, or IRTs) will be reinforced. For example, the experimenter may present reinforcement only for IRT's that exceed 95 percent of the last “n” number of IRT's. The physical value of the criterion IRT consequently is determined by the behavior of the organism. For example, longer IRT's included in the last "n" number of IRT's will result in a longer criterion. If the organism 
continues to emit longer and longer IRT's, the criterion will become longer and longer accordingly. As the behavior of the organism shifts, so does the criterion. In this way, percentile schedules allow the speed of the shaping transition to be determined by the behavior of the individual organism.

\section{Schedule Thinning and Leaning}

In applied settings, treatment of severe problem behavior may involve extremely frequent reinforcer delivery according to one or more schedules, including those delivering the nominal reinforcers independently of responding. These schedules, though effective during the treatment of such behavior, are in some cases impractical to implement over the longer term. Lean schedules often are easier and more practical for caregivers to implement. So, typically an initially rich schedule of reinforcement used at the outset of treatment is leaned or thinned progressively across several sessions until a terminal (and presumably practical, or at least manageable) schedule of reinforcement is in effect (Hagopian, Toole, Long, Bowman, \& Lieving, 2004). There is little doubt that such procedures are effective. Cases in which schedule thinning procedures were effective in maintaining desirable behavior have been reported frequently (e.g., Hagopian et al., 2004; Lalli, Casey, \& Kates, 1997). Questions remain, however, as to the most effective method of thinning.

The crucial question when implementing schedule thinning is how to determine the rate at which reinforcement will decrease. Perhaps the most common method is to determine a fixed amount to decrease the schedule parameter per session. Hagopian, Fisher, and Legacy (1994) found that a fixed-time (FT) 10-s schedule was effective in maintaining low levels of problem behavior in identical quadruplets. The authors then decreased the rate of reinforcement by 1 reinforcer per minute across several sessions. If the programmed rate of reinforcement was 5 
reinforcers per minute for one session, for example, then it was 4 reinforcers per minute in the next session. Then, reinforcement rate continued to decrease across successive sessions in this manner until a terminal FT 5-min schedule was reached. Systematic schedule thinning resulted in the leaned schedule effectively controlling low levels of destructive behavior. This method, however, was far from perfect. For one of the subjects, recurrence of destructive behavior occurred during the thinning process, and it was necessary to "back up" and implement a more gradual thinning procedure.

An alternative to the fixed-decrease method is to thin the schedule based on previous session performance, usually reflected in previous session interresponse times (IRTs). This approach is termed the adjusting- IRT method of schedule thinning and is similar in principle to Terrace's (1963) fading procedure described above. Vollmer, Iwata, Zarcone, and Smith (1993) examined an adjusting procedure for maintaining low levels of attention-maintained selfinjurious behavior (SIB) under differential-reinforcement-of-other-behavior (DRO) schedules. The authors calculated the DRO parameter prior to each session based on mean IRT for a certain number of previous sessions. As the mean IRT for the previous sessions increased, the DRO interval increased accordingly, with the constraint that the schedule could not "back up." That is, the DRO interval could not become shorter even if IRTs became shorter. This procedure was effective in maintaining low rates of SIB throughout schedule thinning.

Although the adjusting method of schedule thinning is similar in some respects to a percentile schedule in the shaping process, it is not a panacea to the problem of determining the schedule thinning rate. Conflicting results have been reported over which method (fixed or adjusting) is more effective. For example, although Vollmer et al. (1993) reported that although an adjusting-increment method was effective, a fixed-increment method was more effective in 
maintaining low rates of SIB during thinning. Alternately, Kahng, Iwata, DeLeon, and Wallace (2000) compared a fixed increment thinning method to an adjusting thinning method and found that with the adjusting IRT method participants reached the terminal schedule faster than with the fixed increment method. The authors point out, however, that the fixed-increment procedure, though based on previous fixed-increment procedures (Hagopian et al. 1994, Vollmer et al. 1993), may not be ideally designed. The authors proposed that other fixed-increment methods of thinning that decrease reinforcement by a fixed percentage rather than a fixed amount may be just as effective as an adjusting-IRT procedure.

Schedule thinning is a useful method in basic research settings as well. For example, schedule thinning in some form or another often appears as an element of pretraining. Few experimenters begin immediately with the baseline schedule parameter following shaping of the target response. Instead, responding initially is reinforced on a richer schedule that is gradually leaned to a terminal (often, baseline) schedule. Laboratory lore suggests that this technique allows the experimenter to "build up" a higher response rate than presumably would be achieved by starting with the baseline schedule parameter. If used successfully, lore has it that this procedure results in relatively higher rates of responding than would be achieved by initial exposure to the baseline schedule alone.

There is little empirical support concerning the effectiveness of the above procedures. Leaning is rarely addressed systematically despite how often it is employed. Both Ferster (1953) and Ferster and Skinner (1957), for example, addressed many aspects of the preparation of subjects for experiments, but made no mention of schedule thinning. One example of the effects of schedule thinning in basic research is the documentation of ratio strain. Ratio strain, or the disruption of responding under increasing ratio schedules, was observed by Ferster and Skinner 
(1957). Increasing the schedule parameter of a ratio schedule too quickly resulted in low, irregular response rates. Increasing the schedule parameter gradually did not result in this disruption. Therefore, it appears that the gradual nature of this procedure is essential for "building up" schedule performance.

Within-session thinning has been investigated in studies that employ progressive schedules. In a progressive ratio schedule, the number of responses that must be emitted to result in reinforcer presentation is increased after each reinforcer delivery. Eventually, the response is extinguished. The ratio size at which extinction occurs is termed the "break point" (Hodos \& Kalman, 1963). Break points appear to be unaffected by the magnitude with which the ratio increases. Stafford and Branch (1998) found that average breaking points on a progressive-ratio schedule were unaffected by step-size magnitude. This does not appear to be the case for progressive-interval schedules, in which reinforcement becomes available contingent upon a response following successive intervals of increasing duration. Dougherty, Cherek, and Roache (1994), for example, found that progressive-interval schedule with smaller step sizes resulted in higher response rates than a progressive interval schedule with larger step sizes.

\section{Controlling Transition Rate}

Across many examples of behavior in transition, it appears that a slow transition is an effective transition, in terms of maintaining behavior across changing conditions. The few examples of leaning, thinning, or fading in the basic literature indicate that the gradual nature of such a procedure often is advantageous in maintaining subsequent responding. But, what is it about gradually changing schedules that allow them to be effective? Two variables determine the rate of a transition. The first is the amount of change, or "step size" between conditions. The second is the frequency with which these changes occur. These variables are often confounded. 
For example, Miller (1960) compared a condition in which shock intensity increased frequently in small steps to a condition in which a large step was presented after a period of stable conditions. Similarly, Terrace (1963) compared a condition in which frequent, small changes in stimulus intensity were compared to a condition in which a large stimulus change was introduced after a period of stable stimulus conditions. Some attempts to consider these variables independently are now considered.

Choice in transition research has investigated the sensitivity of responding to relative rates of reinforcement in concurrent schedules that changed at different frequencies, but with constant step sizes. Gallistel Mark, King, and Latham (2001) investigated rat's lever pressing under concurrent variable-interval (VI) schedules of brain stimulation in which the schedule parameter changed frequently (within session) in one condition and infrequently (between sessions) in another condition. When an unsignaled change in reinforcement rate occurred following a period of schedule stability, responding adjusted slowly to the new schedule conditions. Conversely, when frequent unsignaled schedule changes occurred, responding adjusted more rapidly to the new schedule conditions. In schedule thinning procedures, thinning typically occurs following a period of schedule stability (treatment), and changes in the schedule parameter as thinning usually occur every session or every other session ("infrequently" by Gallistel et al.s' definition). Thus, the ability of schedule thinning procedures to maintain responding may be due to the infrequency with which the schedule parameters change. Corresponding decreases in behavior to decreases in reinforcement rate may take a long time to be evident in behavior, in which case hysteresis may account for the continued elevation of behavior. 
The results of Gallistel et al., however, appear to suggest that response rates eventually adjust to the changed reinforcement rate, even if these changes may require time to take effect. Also, Gallistel et al. used a fluctuating environment in which the concurrent schedule parameters both increased and decreased. In a schedule thinning procedure, changes in the schedule parameter only occur in one direction, although they could change as a function of the performance at any given point in a titrating-type arrangement (e.g. Weiss \& Laties, 1959). It is difficult, however, to anticipate how monotonic changes in reinforcement rate would affect the rate at which new patterns of responding are acquired.

The effects of step size magnitude apart from frequency of change are somewhat more difficult to interpret. Eckerman and colleagues (1980) manipulated transition speed by changing the step size while maintaining a constant frequency of step-size changes, and found that large step sizes were equally effective as small step sizes for shaping key peck location in pigeons. In progressive schedules, the frequency of schedule changes is somewhat regular in that these changes are implemented following reinforcer delivery. The interreinforcer interval increases, however, as the progressive schedule continues to be in effect. Step size magnitude appears to have an effect upon responding is maintained under progressive-interval schedules (Dougherty et al., 1994), but not progressive-ratio schedules (Stafford and Branch, 1998). Further research is invited to clarify the effects of step size changes independently of frequency of change.

\section{Statement of the Problem}

The general goal of a schedule thinning procedure is to maintain behavior despite decreasing reinforcement. Although schedule thinning is generally assumed to be effective in achieving this goal, incidents of failure frequently are observed in the course of the thinning process. For example, it is not uncommon for desirable behavior to drop off occasionally 
throughout schedule thinning, and in some cases it is necessary to "back up" and modify the thinning procedure to accommodate these episodes (e.g., Hagopian et al., 1994). Although schedule thinning may appear to be effective on a large or molar scale, episodes of failure at a more local level may indicate that the procedure is not optimally designed. In some cases, instances in which behavior is not maintained by the thinned schedule may be extremely undesirable. Understanding the conditions under which failure to maintain responding occurs may permit the design and implementation of schedule thinning procedures that reduce or remove these conditions, and thereby allow more effective application of schedule thinning in the numerous and varied settings in which it is used.

The paucity of systematic studies of schedule thinning gives rise to at least two specific questions. The first concerns determining when failure to maintain responding occurs. How might one determine at what point the schedule becomes "too lean" and declare the behavior on the thinned schedule disrupted? Schedule thinning procedures presumably are implemented to prevent the disruptive effects associated with exposure to extinction conditions, most obviously, cessation of the target response. Other disruptive effects, such as behavioral contrast, may be relevant as well. For example, an increase in other concurrently reinforced behavior such as SIB during a schedule thinning process may have important clinical implications. Although extinction and behavioral contrast may indicate failure of the schedule thinning procedure to maintain responding, both measures seldom are employed simultaneously. In applications that use time schedules, an increase in some concurrently reinforced behavior may provide the only index of behavioral sensitivity to schedule thinning. In a basic lab, changes in the rate of the target operant response may be the only indication of the impact of procedural schedule leaning, while other behavior goes unmeasured. 
The second question concerns whether different methods of thinning differ in their behavioral effects. Given the varied research suggesting that gradual environmental changes are more efficacious in maintaining responding, it may be expected that gradual decreases in reinforcement rate will maintain behavior longer than more rapid decreases in reinforcement rate. Although this prediction is supported by cases in which a relatively more gradual schedule thinning procedure was implemented in place of a failed (and relatively more rapid) schedule thinning procedure, different rates of schedule thinning have not been directly compared in terms of their effects on the elimination of the response.

The present study addressed the two questions outlined above. The first was that of at what point in the thinning process the thinned schedule become too lean to maintain behavior in the face of a competing reinforced alternative response. That is, how much of a reduction in programmed reinforcement rate is sufficient to result in behavioral disruption? The present study measured this disruption in two ways. First, disruption was assessed in terms of cessation of responding on the thinned schedule, by assessing the speed and consistency with which responding that resulted in reinforcement presentation according to a thinned schedule was abandoned in favor of responding that resulted in reinforcement according to relatively richer schedule. Additionally, contrast effects as indicated by changes in responding controlled by the richer schedule were taken as additional evidence of disruption of responding in the leaner schedule. The second question concerned whether the rate of thinning would have an effect upon how quickly disruption occurred. In particular, we were interested in whether or not thinning procedures in which reinforcement rate decreases at a constant frequency (daily) but by different magnitudes differ in their ability to maintain behavior. The present study compared the effects of several rates of thinning in terms of their effects on response maintenance. 


\section{Method}

\section{Subjects}

Three male White Carneau pigeons served subjects for the proposed study. Each was individually housed in a home cage with continuous access to water. Each pigeon was maintained at 80 percent of their ad libitum body weight by supplemental feedings with Purina pigeon chow that occurred after experimental sessions as needed. The vivarium was illuminated according to a 12:12 hour light dark cycle. Each pigeon had a history of responding on VI schedules before beginning the present study.

\section{Apparatus}

Two operant conditioning chambers, $32 \mathrm{~cm}$ long by $30 \mathrm{~cm}$ high by $30 \mathrm{~cm}$ wide, each located in a sound-attenuating enclosure were used. An aluminum work panel, comprising one wall of each chamber, contained three $2.54 \mathrm{~cm}$ diameter response keys. Two of the keys in each chamber were transilluminated by a white, green, or red light. The keys were operated by a force of approximately $0.15 \mathrm{~N}$. Reinforcement was $3 \mathrm{~s}$ access to Purina pigeon chow made available from a hopper located behind a $4.5 \mathrm{~cm}$ square feeder aperture located on the midline of the work panel with its lower edge $9 \mathrm{~cm}$ from the chamber floor. During reinforcement presentation, the hopper was raised to be accessible through the aperture, and the aperture was illuminated by a white light. General chamber illumination was provided by a houselight which was on whenever either of the key lights is on, except during reinforcement. White noise and a ventilation fan masked extraneous noise. A personal computer, located in adjacent room, operated medpc7 software which controlled the experiment. 


\section{Procedure}

Daily sessions were conducted at approximately the same time each day. Each session commenced following a 3-min chamber blackout, during which the houselight and all key lights were off. Preliminary training sessions terminated after 60 reinforcer deliveries and sessions during the experiment proper terminated after $60 \mathrm{~min}$. A concurrent schedule of the type described by Findley (1958) was in effect throughout the experiment. During much of the experiment, VI schedules, constructed using 12 intervals arranged as described by Fleshler and Hoffman (1962), were used. The VI schedules were programmed independently of one another; that is, responding in one schedule did not affect the scheduling of reinforcement in the other schedule, and vice versa.

Preliminary training consisted of multiple schedule training followed by switching key training. During multiple schedule training, two VI 10-s components strictly alternated every 3 minutes. During one component, the key was transilluminated green, and during the other component, the key was transilluminated red. Reinforcers programmed but not collected during one VI component were held until the next presentation of the same component. The schedule parameter for both components was increased gradually to a multiple VI 30-s VI 30-s schedule. When responding was stable as judged by visual inspection under these conditions, switchingkey training began.

During switching-key training, two keys were presented. One key (henceforth, the “switching” key) was transilluminated white. Initially, pecks to this key resulted in reinforcer presentations according to a VI 10-s schedule. Additionally, pecks to the switching key changed the color of the other illuminated key (hereafter, the "main" key). Initially, a concurrent VI 30-s VI 30-s schedule was in effect on the main key. Thus, pecks to the switching key allowed access 
to either of the concurrently available schedules on the main key, as described by Findley (1958). After reliable switching was observed, an FR 3 response requirement was instated on the switching key, with the additional requirement that the responses must be consecutive (i.e. emitted sequentially and not interrupted by pecks to the main key) to result in a schedule switch. At this point, reinforcement was no longer provided for pecks to the switching key. Pecks to the main key reset the ratio requirement on the switching key to 3. Completion of the FR requirement changed the color on the main key and was counted as a switch. To prevent rapid switching, a 3-s changeover delay (COD) was initiated immediately following a switch. During the COD, the stimuli did not change, however pecks to the main key did not result in reinforcement presentation. Then, the schedule parameter of the concurrent schedules was increased gradually to VI 120-s VI 120-s across several sessions.

Baseline. After preliminary training was completed, a concurrent schedule was arranged as described by Findley (1958). Two independent VI 120-s schedules were in effect on the main key. Single responses on the change-over key did not result in any programmed stimulus change. The key color on the main key correlated with either of the VI schedules (red or green) that was in effect at the beginning of the session was selected randomly before each session. The baseline condition was in effect for a minimum of 30 sessions and until responding in each component was stable as judged by visual inspection, but for no more than 50 total sessions.

Schedule Thinning and Extinction. Table 1 shows the sequence of conditions for each pigeon. Responding was observed under three schedule thinning conditions and one extinction condition. These conditions were separated by a return to the baseline condition, as seen in Table 1. In the schedule thinning conditions, the mean interval of one of the concurrently available schedules was increased by 10,20 , or 70 percent across sessions, thereby decreasing 
the programmed reinforcement rate for that schedule. For example, during $20 \%$ thinning the mean interval of one of the schedules was increased by $20 \%$ per session (i.e., the length of each of the intervals comprising the VI distribution increased by $20 \%$ ). In all thinning conditions, the other concurrently presented schedule remained VI 120-s. Thinning was in effect until the criterion for extinction was met in the relatively leaner schedule component (i.e., the pigeon allocated no more than $5 \%$ of the session duration to the leaned schedule).

During the extinction condition, responses in the presence of one of the stimuli did not result in reinforcement presentation and responses in the presence of the other stimulus resulted in reinforcement presentation according to a VI 120-s schedule. Extinction was in effect until the criterion for extinction was met in the extinction component. Table 1 shows the stimuli that were correlated with thinned schedule or extinction for these conditions. As seen in Table 1, the order of exposure to $10 \%$ and $20 \%$ thinning conditions were counterbalanced, and the order of exposure to $70 \%$ and extinction conditions were counterbalanced across subjects. The stimuli correlated with the reduced reinforcement schedule were alternated across thinning and extinction conditions.

\section{Results}

Figure 1 shows the time allocated to each of the component schedules across conditions. Because the experimental arrangement dictated that responding occur in the presence of the green keylight or red keylight, as time allocated to one of the components decreased, time allocated to the other schedule necessarily increased. This is shown by the symmetrical data paths in Figure 1. During baseline sessions, Pigeons 567 and 609 allocated approximately half of the session (i.e., 30 minutes) to each of the component schedules. Pigeon 775 consistently allocated more time to the schedule correlated with the red keylight across all baseline sessions. 
For all subjects, time allocated to the thinned component decreased as the schedule was thinned in all thinning conditions, as well as the extinction condition. After the baseline schedules were reinstated, responding rapidly returned to a level comparable to that observed in the preceding baseline condition, usually within about 5 sessions.

Figure 2 shows the overall response rates (total number of responses divided by session duration less reinforcement time, i.e., 60 minutes) in each of the component schedules for sessions across conditions. For all subjects, rate of responding in the thinned component decreased as the schedule was thinned or when extinction was in effect. Rate of responding increased in the not-thinned component during schedule thinning and extinction, despite programmed reinforcement rate remaining constant in that schedule. Response rates in the notthinned schedule generally increased to the same level during all experimental conditions before the criterion for extinction was met. A general increase in both responding and time allocation was observed in the not-thinned component during all experimental phases for all subjects. Thus, similar contrast effects were observed in the not-thinned schedule in both response rate and time allocation measures during schedule thinning and extinction conditions.

As seen in Figure 3, obtained reinforcement rates (total number of reinforcers divided by session duration less reinforcement time) were generally equivalent across components during baseline sessions. Obtained reinforcement rates in the thinned component decreased across sessions of thinning. When the baseline conditions were reinstated following a thinning or extinction phase, obtained reinforcement rates in the thinned component quickly returned to baseline levels. During thinning, obtained reinforcement rates in the not-thinned component generally increased moderately, most likely as a result of the increase in time allocation to that component. 
Figure 4 shows the programmed and obtained reinforcement rates in the thinned component for all thinning conditions. Obtained reinforcement rate was approximately equal to or slightly less than programmed reinforcement rate in all thinning conditions. More gradual thinning resulted in higher reinforcement rates throughout the thinning process relative to more rapid thinning conditions. Responding was eliminated (and the thinning condition was terminated) when obtained reinforcement rate reached zero or near-zero levels in all conditions. In Figure 5, the obtained reinforcement rate functions from Figure 4 are shown with linear trend lines. The slopes and intercepts of the linear trend line for these functions were not consistently different across thinning conditions.

Figure 6 shows response rates in the thinned component relative to the mean response rate for the last 6 sessions of the preceding baseline for all thinning conditions. More gradual thinning conditions maintained responding for a greater number of sessions than more rapid thinning conditions. In general, the more gradually the schedule was thinned, the greater the relative response rate maintained across sessions of thinning. One exception is for 567's extinction condition, which maintained a higher proportion of baseline responding than the 70 percent thinning condition. The slope of the linear trend line was generally steeper for the more rapid thinning conditions relative to more gradual thinning conditions.

Figure 7 shows the log-transformed response ratios (response rate for the constant schedule divided by response rate for the thinned schedule) as a function of the log-transformed reinforcer ratios (reinforcement rate for the constant schedule divided by reinforcement rate for the thinned schedule) for each session of each thinning condition for all subjects (note: 0.01 was added to each outcome to avoid division by zero in ratio calculation). The generalized matching equation (Equation 1, shown below; Baum, 1974) was fitted using the least-squares method. 


$$
\log \left(\frac{R_{1}}{R_{2}}\right)=s \cdot \log \left(\frac{R f_{1}}{R f_{2}}\right)+\log (b)
$$

$R_{1}$ and $R_{2}$ identify response rates for the constant and thinned schedules, respectively. $R f_{1}$ and $R f_{2}$ identify reinforcement rates for the constant and thinned schedules, respectively. The parameter $b$ identifies bias, in which a positive value indicates bias for the constant schedule (i.e., intercept $<0$ ). The parameter $s$ identifies sensitivity, in which a value $>1$ indicates overmatching, or more extreme response allocation. A value of $s<1$ indicates undermatching, or less extreme response allocation. The thick line represents strict matching $(b=0, s=1)$. Table 2 shows the bias, sensitivity, and variance accounted for by generalized matching across all thinning conditions.

As previously stated, the rationale of schedule thinning implies that reducing reinforcement gradually will maintain behavior more effectively than reducing reinforcement rapidly. In terms of a matching analysis, this implies that organisms undermatch when a schedule is thinned (i.e., continue to allocate responding to a schedule despite decreased reinforcement provided by that schedule). In contrast, pigeons generally matched response allocation to relative rates of reinforcement provided by the schedules, regardless of the rate at which reinforcement was decreased for one schedule. This implies that rate of responding controlled by a schedule will be similar regardless of the number of steps of thinning required to reach that schedule.

\section{Discussion}

As noted in the statement of the problem, the present study addressed two questions. The first question was that of determining when in the thinning process disruption of behavior occurs. Two indicators of disruption were examined: reduced responding on the thinned schedule and increased responding on the constant (i.e., not- thinned) schedule. Across all thinning 
conditions, responding on the thinned schedule and time allocated to that schedule decreased across sessions as the schedule was thinned. These changes were accompanied by corresponding increases in time allocation and response rate to the constant schedule. Thus, both measures indicated that disruption was occurring on a session-by-session basis. The second question was whether different rates of thinning would differ in their behavioral effects. The rate of thinning was controlled by manipulating the step size (percentage change) of the thinning transition, with a consistent frequency of change (each session). Relatively more rapid schedule thinning, resulting from a larger step size, resulted in more rapid decrements in response rate and time allocation to the thinned schedule. Such rapid thinning also resulted in more rapid increases in time allocation and response rate to the constant schedule.

This experiment was, to the author's knowledge, the first systematic investigation of different rates of schedule thinning in which frequency of change was constant throughout thinning. In the few prior studies that investigated different methods of thinning, the same thinning rate was used for all participants (e.g., Hagopian et al., 1994), frequency of change was different for each participant (Vollmer et al., 1993; Kahng et al., 2012), or different rates of thinning involved different frequencies of change as well as differing step sizes (Hagopian et al., 2004). Thinning the schedule with a constant frequency of change allowed the effects of several different step sizes to be assessed.

In the following sections, I will discuss qualifications and potential limitations of the present research. Then, I will discuss the present study in relation to previous research on extinction, schedule thinning, and behavioral transitions, generally. Finally, some implications of the present study for applied research are suggested. 


\section{Qualifications of the Present Research}

As with any repeated-measures research design, the design of the present study involved the risk of history effects. Subjects were exposed repeatedly to thinning conditions throughout the experiment, and it is possible that exposure to thinning in prior experimental conditions could have affected performance during subsequent thinning conditions differently than if those prior thinning conditions had not been conducted. The question of what effects, if any, repeated exposure to thinning has on response elimination is yet to be addressed.

Schedule thinning and extinction in the present study both involved a transition to no reinforcement. The schedule thinning condition and the extinction condition were distinguished by the speed at which this transition was imposed. If schedule thinning is conceptualized as "slow extinction" (i.e., a more graded version of the transition to no reinforcement), then one might expect repeated exposure to thinning to resemble repeated exposure to extinction. Anger and Anger (1976) investigated repeated exposure to extinction by alternating 2-session periods of reinforcement for keypecking with 8-session periods of extinction for as many as 12 cycles. During the first extinction condition, response rates decreased across successive sessions. After several extinction cycles, response rates during the first session of an extinction condition were lower relative to the first session of prior extinction conditions. After approximately 8 cycles of response retraining followed by extinction, response rates were at near-zero levels as early as the first extinction session. Generally, repeated exposure to extinction resulted in more rapid response elimination in subsequent extinction conditions. Thus, if schedule thinning resembles extinction studied in this way, more rapid response elimination would be expected with repeated exposure to thinning. 
The potential confound of repeated exposure to thinning was addressed by instating a session minimum for baseline stability, on the assumption that a sufficiently long (i.e., greater than 30 day) baseline condition between thinning conditions might attenuate this effect, if present, or at least minimize it. In the previously described Anger and Anger (1976) study, only 2 days of reinforcement intervened between successive extinction conditions. If 1) repeated exposure to thinning results in more rapid response elimination, and 2) the 30 -session minimum was ineffective in attenuating this effect, more rapid response elimination might be expected with each successive exposure to thinning, regardless of the thinning rate. This may have been the case for Pigeon 567, which was exposed to thinning conditions in an escalating order. Both Pigeons 775 and 609, however, exhibited more rapid response elimination under the extinction condition relative to the $70 \%$ thinning condition, despite this thinning condition occurring after the extinction condition.

Any within-subjects parametric investigation poses the risk of order effects. In the present study, multiple transitions to no reinforcement were conducted in the presence of each of the key colors. Thinning in the presence of a key color that was previously correlated with a faster transition (i.e., a relatively more rapid thinning condition or extinction) might result in more rapid response elimination than would be observed in the absence of such a history. However, no systematic effects as a function of key color were observed. For example, Pigeons 775 and 609 both were exposed to extinction in green. The green keylight was previously correlated with $10 \%$ thinning for 609 , and with $20 \%$ thinning for 775 . Despite a differential history with the green key light, extinction resulted in similar rates of response elimination for both subjects. The present data, therefore, do not suggest that behavior in the thinning or 
extinction conditions was under the control of variables other than the contingencies in effect in those conditions.

The concurrent schedule, as noted above, allowed assessment of thinning effects on both the target behavior and on other behavior for which the contingencies remained unchanged. As with any concurrent schedule, response rates for each of the components can be calculated in two ways, which may lead to different conclusions about the data. Here, response rate for each component in a given session was calculated by dividing the total responses on one schedule by the total session time (i.e., $60 \mathrm{~min}$ ). Using this method, response rates for each component are essentially half the overall response rate (excluding responses on the changeover key). Thus, response rates for each component schedule were low, even though the overall session response rate might be reasonably high. An alternative is to calculate response rates for each component individually by dividing the total number of responses in a component by time allocated to that component. Both methods of calculating response rates yield similar results during baseline conditions, when time allocated to both schedules is approximately equal (i.e., $30 \mathrm{~min}$, See Figure 1). When the thinning and extinction conditions are considered, however, these methods lead to different conclusions regarding effects of the transition to no reinforcement on responding on 1) the constant and 2) the reduced-reinforcement schedule that will now be considered.

Absolute response rate in the constant schedule increased as the schedule was thinned, and this effect was labeled a contrast effect. When response rates relative to time allocation are examined, however, responding in the constant schedule does not increase. Pigeons did not respond differentially faster in the presence of the stimulus correlated with the constant schedule during thinning and extinction, but rather allocated more of the session time to that schedule. 
The contrast effect observed in the present experiment is therefore a result of increased time allocation to the constant schedule.

When response rates relative to time allocation (i.e., responses on Key A/time spent in the presence of Key A) are calculated for the thinning and extinction conditions of the present study, the denominator of this calculation decreases across the condition. This is because time allocated to the reduced-reinforcement schedule decreases across the condition (see Figure 1). Thus, the response rate on the reduced-reinforcement schedule is inflated. This is especially true during extinction, in which response rates dramatically increased during some extinction sessions using this method of calculation. When absolute response rates are calculated, the denominator of the rate calculation remains constant across the condition (i.e., $30 \mathrm{~min}$ ). This method results in response elimination functions that show progressive decreases in responding across sessions in thinned schedule. The absolute response rate method therefore provides a more precise description of the extinction process. It was this reason that it was chosen as the primary mode of data presentation for the present study.

\section{Relation to Previous Research}

Extinction. Introducing extinction gradually via schedule thinning did increase the number of sessions required to eliminate responding, and did result in higher levels of behavior throughout the transition to extinction. In this way, the response elimination functions resemble behavior that is more "resistant" to extinction, as resistance to extinction has been discussed previously in the literature. However, this increased "resistance" can be explained by the increased number of sessions required to eliminate reinforcement at a more gradual rate. Behavior persisted on the lean schedule only to the extent to which reinforcement persisted on that schedule, regardless of the rate of thinning. There was no added benefit in terms of behavior 
maintenance in proceeding more slowly in the transition to extinction. Moreover, the process of thinning as arranged here did not produce effects that were markedly different from conventional extinction in terms of response elimination, except for the speed at which these transitions occurred. That is, the effects of different rates of decreasing reinforcement did not differ qualitatively, although they sometimes did differ quantitatively. Both thinning and extinction produce declining response elimination functions, and the differences between conditions were only in the slope of these functions.

This is consistent with the broad literature that pigeons match their responding to rate of reinforcement provided by a schedule (see Pierce \& Epling, 1983, for a review), even when reinforcement rates fluctuate rapidly (e.g., Gallistel et al., 2001), but extends this finding to conditions with progressively decreasing rates of reinforcement. Previous investigations of schedule thinning did not report effects of thinning on response elimination, so it is impossible to determine if these results are inconsistent with other schedule thinning procedures. The rationale of schedule thinning, however, implies that when a schedule is gradually thinned the organism will persist in responding despite decreased reinforcement. In a matching analysis, this would be expressed in undermatching. To the contrary, these results show that pigeons matched, regardless of the speed of the thinning.

Two other extinction effects were discussed in the literature review: behavioral contrast and resurgence. In the treatment of problem behavior, practitioners implement thinning also (or primarily) to avoid either or both of these effects. The appearance of problem behavior during thinning could be a resurgence effect (if the problem behavior is currently under extinction) or a behavioral contrast effect (if the problem behavior is concurrently reinforced). Winterbauer and Bouton (2010) found that resurgence was reduced when reinforcement was thinned to extinction, 
relative to an immediate extinction condition. These results were replicated by Sweeney and Shahan (2013). Both findings indicate that thinning can reduce extinction-like effects on other behavior. By comparison, our findings did not show any difference in behavioral contrast as a result of thinning. Thus, it is unclear whether thinning will be able to attenuate extinction-like effects on other behavior, and if thinning is more effective at attenuating certain effects than others.

\section{Schedule Thinning and Leaning}

Applied Methods of Schedule Thinning. In applied contexts, schedule thinning is a tool for decreasing reinforcement to a more manageable level, or "practical" schedule. Thus, it is unlikely that a practitioner would thin the schedule all the way to extinction (as was the case in this experiment). Although the present study did not stop thinning at a leaner schedule, the matching analysis showed that roughly equivalent rates of responding were maintained by roughly equivalent rates of reinforcement, even if the transition to the lower level of reinforcement took many more steps. Thus, it appears that transitioning more gradually to a leaner schedule does not have any added value in terms of behavior maintenance. This is consistent with the findings of Hagopian et al. (2004), which demonstrated that an abrupt transition to a leaner schedule was equally as effective as a gradual transition (though the "effectiveness" was judged in terms of the absence of problem behavior rather than behavior maintained by the lean schedule).

Progressive Schedules. Progressive-interval (PI) schedules are essentially withinsession schedule thinning, in which the schedule is thinned (i.e., interval increased) following each reinforcer. The step size of the transition is the amount by which the interval is increased. Dougherty, Cherek, and Roache (1994) investigated the effects of step size on progressive- 
interval performance, and found that smaller step sizes resulted in higher rates of responding than PI schedules with larger step sizes. This result is consistent with the findings of the present experiment, in which smaller step sizes resulted in higher rates of responding throughout thinning. Dougherty et al. did not keep the PI schedule in place until responding was eliminated, so it is impossible to say whether the higher rates of responding observed were simply a function of increased reinforcement on the small step size PI, like what was observed in the present experiment.

The results are consistent with response elimination effects observed in progressive ratio (PR) schedules. Increasing step size does not affect the "break point," or schedule value at which responding is eliminated, in a progressive ratio schedule (Stafford \& Branch, 1998). Similarly, response elimination was observed when obtained reinforcement rate reached zero regardless of step size in the present study.

\section{Controlling Transition Rate}

As discussed in the literature review, the two variables that control the rate of a transition (frequency of change and step size) are often confounded in previous comparisons of gradual versus rapid transitions. One investigation that controlled for frequency of change investigated shaping with different step sizes. Eckerman and colleagues (1980) found that, when frequency of change was held constant, there was no effect of step size on behavior observed at the final performance criterion. This is consistent with the present results.

\section{Implications for Application}

As previously discussed, the process of thinning as arranged here did not produce effects that were markedly different from conventional extinction in terms of response elimination, except for the speed at which these transitions occurred. Both thinning and extinction produce 
declining response elimination functions, and the differences between conditions were only in the slope of these functions. An applied researcher would rarely (if ever) thin a schedule all the way to extinction, because usually the goal is merely a leaner schedule of reinforcement. It is perhaps important to note, however, that decreasing reinforcement is likely to produce the extinction-like effects observed here, even if these effects progress more slowly.

When thinning a schedule, one should expect increased time and responding allocated to other schedules of reinforcement that are concurrently available. This may be especially relevant in contexts which present relatively sparse environments with few options for behavioral variability, or in cases in which two mutually exclusive behaviors provide access to the same reinforcer, as was the case in the present study. For example, consider that a functionally equivalent response (e.g., a student requesting teacher attention while at their desk) has been trained to replace a problem behavior (e.g., elopement that was attention maintained). These responses are mutually exclusive, and provide access to the same reinforcer. If the schedule of attention for requests is thinned, one should be prepared for increases in elopement. However, these increases would be more gradual (and, perhaps, manageable) if a more gradual rate of thinning is employed.

Thinning, even at a very rapid pace, maintains responding more effectively than conventional extinction. Two of three pigeons in the present study continued to respond on the thinned schedule for several sessions, even when the decreases in reinforcement were severe (70\% per session). That suggests that, if necessary, some thinning (even thinning that is implemented at an extremely rapid pace) may be better than no thinning at all (i.e., extinction) if the goal is to sustain behavior as long as possible. If, however, the goal is to eliminate behavior quickly, conventional extinction is the better alternative. 


\section{Future Directions}

In the introduction, it was observed that schedule thinning is often done, but less often understood. This study addressed this need by contributing to the relatively limited literature on schedule thinning. As is the case with any contribution to scientific inquiry, however, these findings raise questions in addition to answering them. Some particularly inviting areas of future research will now be considered.

Systematic differences in response elimination as a function of thinning rate were not observed. Earlier it was noted that schedule thinning procedures are evaluated in terms of responding other than responding for which reinforcement is being thinned. For example, failure of schedule thinning may refer to an increase in problem behavior. Thus, an experimental analysis of schedule thinning effects might also focus on other behavior as an indication of schedule thinning effects. One might extend the findings of Winterbauer and Bouton (2010) and Sweeney and Shahan (2013), by examining the effects of different rates of thinning on resurgence. Alternately, schedule thinning could be compared to extinction in terms of extinction-induced aggression (e.g., Azrin, Hutchinson, \& Hake, 1966).

Because step size does not appear to affect thinning when frequency of change is controlled for, future research might incorporate frequency of change into an analysis of thinning. For example, a future study may investigate whether changes in reinforcement rate using large step sizes maintains behavior more effectively than smaller ones if the large step size changes are implemented relatively less frequently than the small step size changes. For example, consider a thinning procedure (A) in which reinforcement is reduced by 1 reinforcer each session, and a different procedure (B) in which reinforcement is reduced by 2 reinforcers, but only every other session. Both A and B result in the same overall rate of thinning, but would 
one of them be more effective in maintaining responding? Procedure B might be a more attractive option for schedule thinning implementers because it requires programming half the transitions of Procedure A. But if Procedure B does not produce least comparable maintenance of behavior relative to Procedure A, schedule thinning implementers might prefer the relatively more planning-intensive Procedure A.

Another area of research that is particularly relevant from an applied perspective is the comparison of ratio thinning and interval thinning, because many applications involve thinning from an FR 1 (i.e., continuous reinforcement) schedule. In this discussion, ratio and interval thinning were not discussed as conceptually distinct processes. These methods may lead to different questions in terms of how to design an effective thinning procedure. In interval thinning, the question is how to maintain behavior with less reinforcement. The question in ratio thinning is how to get more behavior for the same reinforcement, rather than decreasing reinforcement per se. Thus, further research is invited to clarify to what degree generalization among these two types of thinning is valid.

\section{Conclusion}

The transition between rich and lean reinforcement is a common one, perhaps most notably in the reinforcement-based treatment of problem behavior. Many reinforcement-based treatments involve schedules of reinforcement that are too rich to continue to implement longterm, thus, treatment often necessitates schedule thinning. Understanding which variables result in successful thinning is therefore critical for ensuring the long-term success of reinforcementbased treatment. Thinning reinforcement by changing the step size of does not result in differential behavior maintenance by a lean schedule. If the goal is to maintain responding with 
a lean schedule, this study suggests that an abrupt transition to that leaner schedule is equally effective as a gradual transition. 


\section{References}

Anger, D., \& Anger, K. (1976). Behavior changes during repeated eight-day extinctions. Journal Of The Experimental Analysis Of Behavior, 26(2), 181-190. doi:10.1901/jeab.1976.26-181

Azrin, N. H., Hutchinson, R. R., \& Hake, D. F. (1966). Extinction-induced aggression. Journal Of The Experimental Analysis Of Behavior, 9(3), 191-204. doi:10.1901/jeab.1966.9-191

Baum, W. M. (1974). On two types of deviation from the matching law: Bias and undermatching. Journal Of The Experimental Analysis Of Behavior, 22(1), 231-242. doi:10.1901/jeab.1974.22-231

Dougherty, D. M., Cherek, D. R., \& Roache, J. D. (1994). The effects of smoked marijuana on progressive-interval schedule performance in humans. Journal Of The Experimental Analysis Of Behavior, 62(1), 73-87. doi:10.1901/jeab.1994.62-73

Eckerman, D. A., Hienz, R. D., Stern, S., \& Kowlowitz, V. (1980). Shaping the location of a pigeon's peck: Effect of rate and size of shaping steps. Journal Of The Experimental Analysis Of Behavior, 33(3), 299-310. doi:10.1901/jeab.1980.33-299

Ferster, C. B. (1953). The use of the free operant in the analysis of behavior. Psychological Bulletin, 50(4), 263-274. doi:10.1037/h0055514

Ferster, C. B., \& Skinner, B. F. (1957). Schedules of reinforcement. East Norwalk, CT US: Appleton-Century-Crofts. doi:10.1037/10627-000

Findley, J. D. (1958). Preference and switching under concurrent scheduling. Journal Of The Experimental Analysis Of Behavior, 1123-144. doi:10.1901/jeab.1958.1-123

Fleshler, M., \& Hoffman, H. S. (1962). A progression for generating variable-interval schedules. Journal Of The Experimental Analysis Of Behavior, 5(4), 529-530. doi:10.1901/jeab.1962.5-529 
Galbicka, G. (1994). Shaping in the 21st century: Moving percentile schedules into applied settings. Journal Of Applied Behavior Analysis, 27(4), 739-760. doi:10.1901/jaba.1994.27-739

Gallistel, C. R., Mark, T. A., King, A., \& Latham, P. E. (2001). The rat approximates an ideal detector of changes in rates of reward: Implications for the law of effect. Journal Of Experimental Psychology: Animal Behavior Processes, 27(4), 354-372. doi:10.1037/0097-7403.27.4.354

Hagopian, L. P., Fisher, W. W., \& Legacy, S. M. (1994). Schedule effects of noncontingent reinforcement on attention-maintained destructive behavior in identical quadruplets. Journal Of Applied Behavior Analysis, 27(2), 317-325. doi:10.1901/jaba.1994.27-317

Hagopian, L. P., Toole, L. M., Long, E. S., Bowman, L. G., \& Lieving, G. A. (2004). A comparison of dense-to-lean arid fixed lean schedules of alternative reinforcement and extinction. Journal Of Applied Behavior Analysis, 37(3), 323-337. doi:10.1901/jaba.2004.37-323

Hodos, W., \& Kalman, G. (1963). Effects of increment size and reinforcer volume on progressive ratio performance. Journal Of The Experimental Analysis Of Behavior, 6(3), 387-392. doi:10.1901/jeab.1963.6-387

Kahng, S., Iwata, B. A., DeLeon, I. G., \& Wallace, M. D. (2000). A comparison of procedures for programming noncontingent reinforcement schedules. Journal Of Applied Behavior Analysis, 33(2), 223-231. doi:10.1901/jaba.2000.33-223

Karsh, E. B. (1963). Changes in intensity of punishment: Effect on running behavior of rats. Science, 140(Whole No. 3571), 1084-1085. doi:10.1126/science.140.3571.1084 
Lalli, J., Casey, S. D., \& Kates, K. (1997). Noncontingent reinforcement as treatment for severe problem behavior: Some procedural variations. Journal Of Applied Behavior Analysis, 30(1), 127-137. doi:10.1901/jaba.1997.30-127

Lattal, K. A., \& St. Peter Pipkin, C. (2009). Resurgence of previously reinforced responding: Research and application. The Behavior Analyst Today, 10(2), 254-266.

Miller, N. E. (1960). Learning resistance to pain and fear: Effects of overlearning, exposure, and rewarded exposure in context. Journal Of Experimental Psychology, 60(3), 137-145. doi:10.1037/h0043321

Nevin, J. A. (1974). Response strength in multiple schedules. Journal Of The Experimental Analysis Of Behavior, 21(3), 389-408. doi:10.1901/jeab.1974.21-389

Nevin, J. A. (1988). Behavioral momentum and the partial reinforcement effect. Psychological Bulletin, 103(1), 44-56. doi:10.1037/0033-2909.103.1.44

Pierce, W., \& Epling, W. (1983). Choice, matching, and human behavior: A review of the literature. The Behavior Analyst, 6(1), 57-76.

Reynolds, G. S. (1961). Behavioral contrast. Journal Of The Experimental Analysis Of Behavior, 457-71. doi:10.1901/jeab.1961.4-57

Stafford, D., \& Branch, M. N. (1998). Effects of step size and break-point criterion on progressive-ratio performance. Journal Of The Experimental Analysis Of Behavior, 70(2), 123-138. doi:10.1901/jeab.1998.70-123

Sweeney, M. M., \& Shahan, T. A. (2013). Effects of high, low, and thinning rates of alternative reinforcement on response elimination and resurgence. Journal Of The Experimental Analysis Of Behavior, 100(1), 102-116. doi:10.1002/jeab.26 
Terrace, H. S. (1963). Discrimination learning with and without 'errors.'. Journal Of The Experimental Analysis Of Behavior, 6(1), 1-27. doi:10.1901/jeab.1963.6-1

Vollmer, T. R., Iwata, B. A., Zarcone, J. R., \& Smith, R. G. (1993). The role of attention in the treatment of attention-maintained self-injurious behavior: Noncontingent reinforcement and differential reinforcement of other behavior. Journal Of Applied Behavior Analysis, 26(1), 9-21. doi:10.1901/jaba.1993.26-9

Weiss, B., \& Laties, V. G. (1959). Titration behavior on various fractional escape programs. Journal Of The Experimental Analysis Of Behavior, 2227-248. doi:10.1901/jeab.1959.2227

Winterbauer, N. E., \& Bouton, M. E. (2012). Effects of thinning the rate at which the alternative behavior is reinforced on resurgence of an extinguished instrumental response. Journal Of Experimental Psychology: Animal Behavior Processes, 38(3), 279-291. doi: $10.1037 / \mathrm{a} 0028853$ 
Table 1.

Sequence of Conditions

\section{Pigeon ID}

$\begin{array}{llll} & 775 & 609 & 567 \\ 1 & \text { BL (50) } & \text { BL (34) } & \text { BL (48) } \\ 3 & 20 \% \text { in Green (16) } & 10 \% \text { in Green (30) } & 10 \% \text { in Red (19) } \\ 4 & \text { BL (50) } & \text { BL (31) } & \text { BL (32) } \\ 5 & 10 \% \text { in Red (15) } & 20 \% \text { in Red (22) } & 20 \% \text { in Green (15) } \\ 6 & \text { BL (50) } & \text { BL (50) } & \text { BL (50) } \\ 8 & \text { EXT in Green (7) } & \text { EXT in Green (6) } & 70 \% \text { in Red (6) } \\ 7 & \text { BL (50) } & \text { BL (50) } & \text { BL (50) } \\ & 70 \% \text { in Red (10) } & 70 \% \text { in Red (10) } & \text { EXT in Green (7) }\end{array}$

Note. BL and EXT identify baseline and extinction conditions, respectively. The number of sessions per condition are identified within parentheses. 
Table 2

Parameters and Variance Accounted for by the Generalized Matching Equation

\begin{tabular}{|c|c|c|c|c|c|c|c|c|c|}
\hline \multirow[b]{2}{*}{ Pigeon } & \multicolumn{3}{|c|}{$\operatorname{Bias}(b)$} & \multicolumn{3}{|c|}{ Sensitivity $(s)$} & \multicolumn{3}{|c|}{ Variance $\left(\mathrm{R}^{2}\right)$} \\
\hline & 10 & 20 & 70 & 10 & 20 & 70 & 10 & 20 & 70 \\
\hline 567 & 0.40 & 0.66 & 1.17 & 0.58 & 0.79 & 0.66 & 0.82 & 0.62 & 0.81 \\
\hline 609 & 0.17 & 0.44 & 0.51 & 0.98 & 1.19 & 1.49 & 0.72 & 0.57 & 0.65 \\
\hline 775 & -0.73 & 1.04 & -1.06 & 0.91 & 0.91 & 1.88 & 0.96 & 0.77 & 0.75 \\
\hline
\end{tabular}

Note. 10,20 , and 70 represent $10 \%, 20 \%$, and $70 \%$ thinning conditions, respectively. 


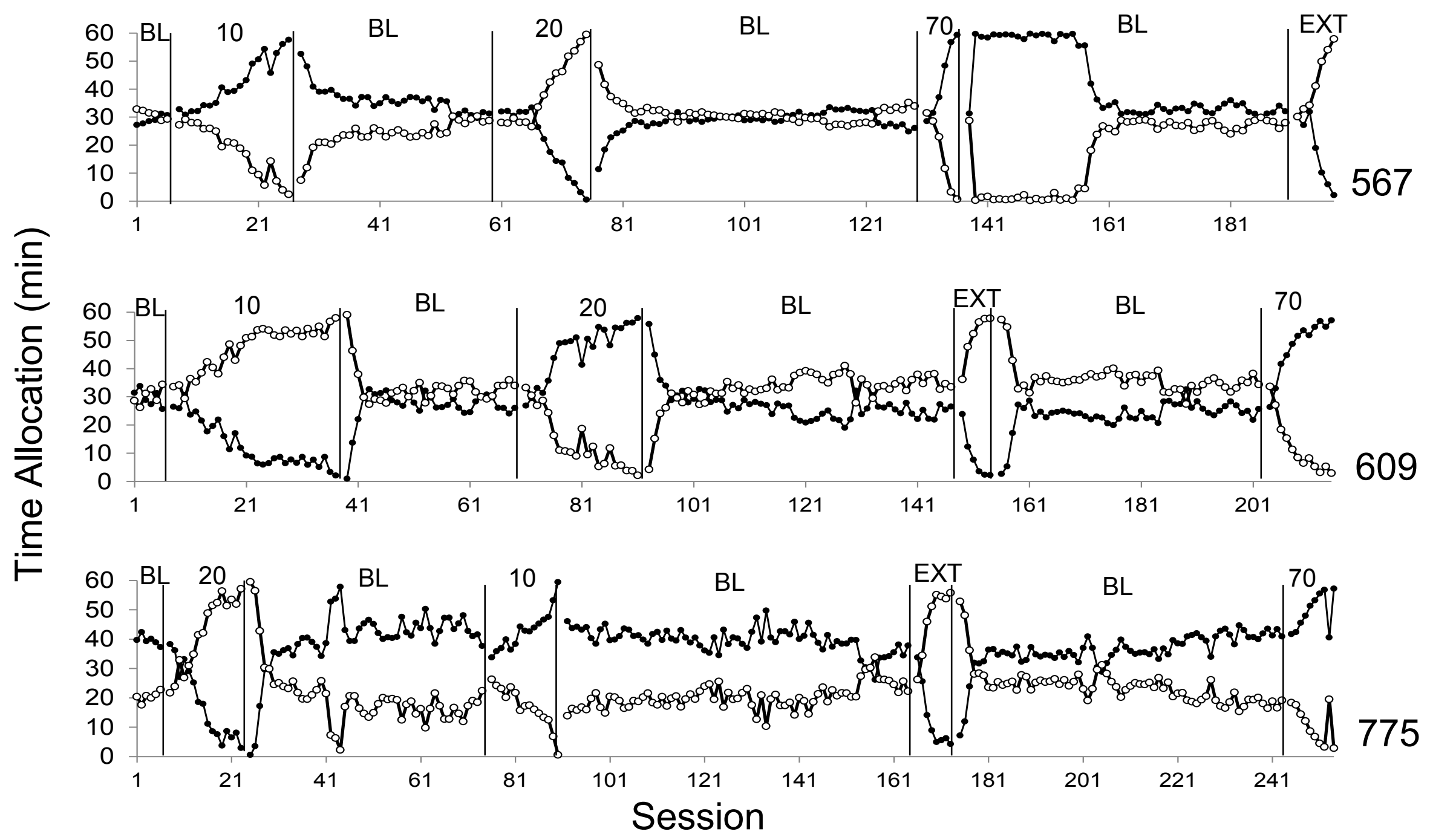

Figure 1. Time allocated in minutes to each of the component schedules for the last 6 session s of the first baseline and subsequent experimental conditions. Filled data points represent the schedule correlated with green, open data points represent the schedule correlated with red. BL and EXT represent baseline and extinction, respectively. 10, 20, and 70 represent 10\%, 20\%, and 70\% thinning conditions, respectively. 

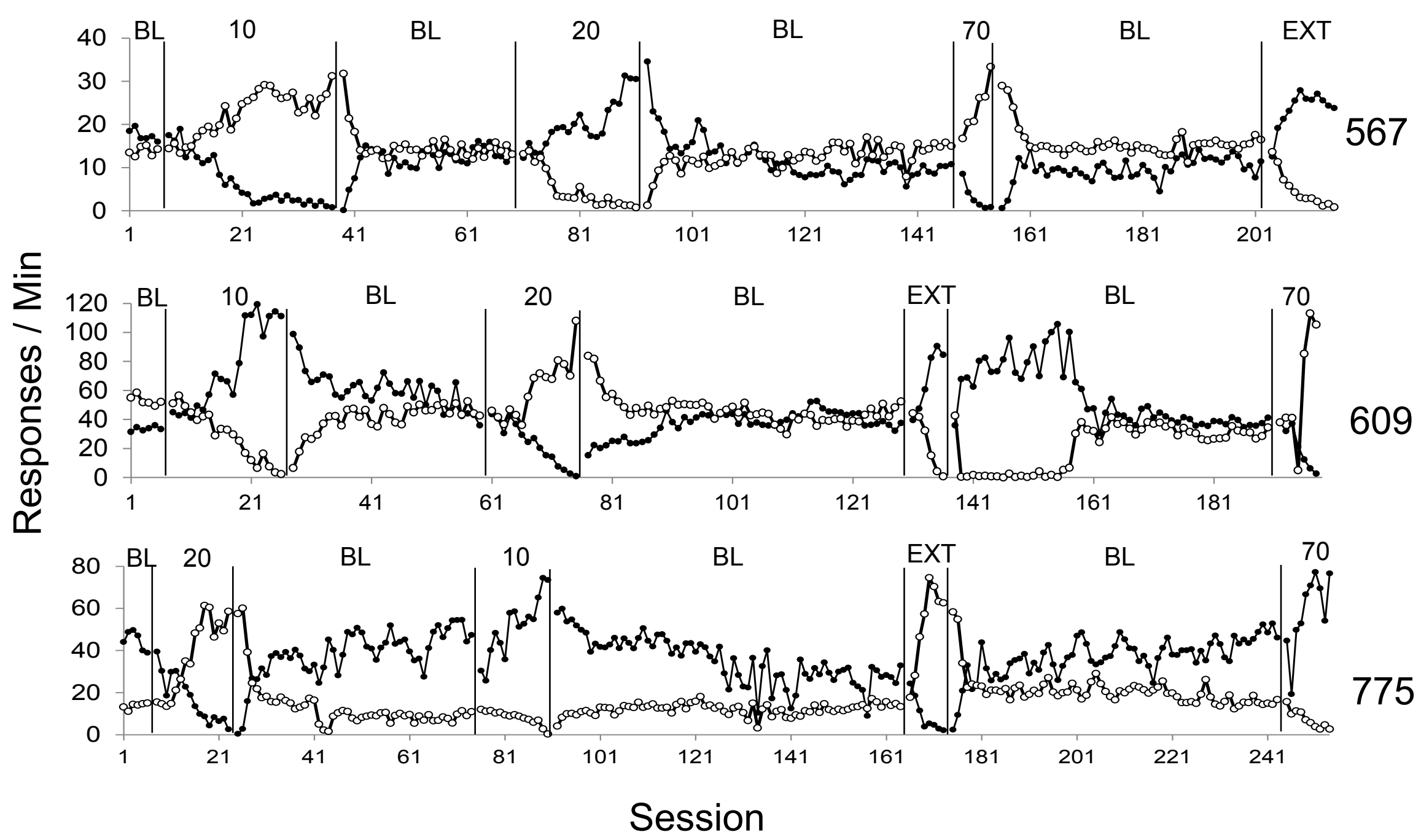

Figure 2. Response rates for each of the component schedules for the last 6 session $\mathrm{s}$ of the first baseline and subsequent experimental conditions. Filled data points represent the schedule correlated with green, open data points represent the schedule correlated with red. BL and EXT represent baseline and extinction, respectively. 10, 20, and 70 represent $10 \%, 20 \%$, and $70 \%$ thinning conditions, respectively. 

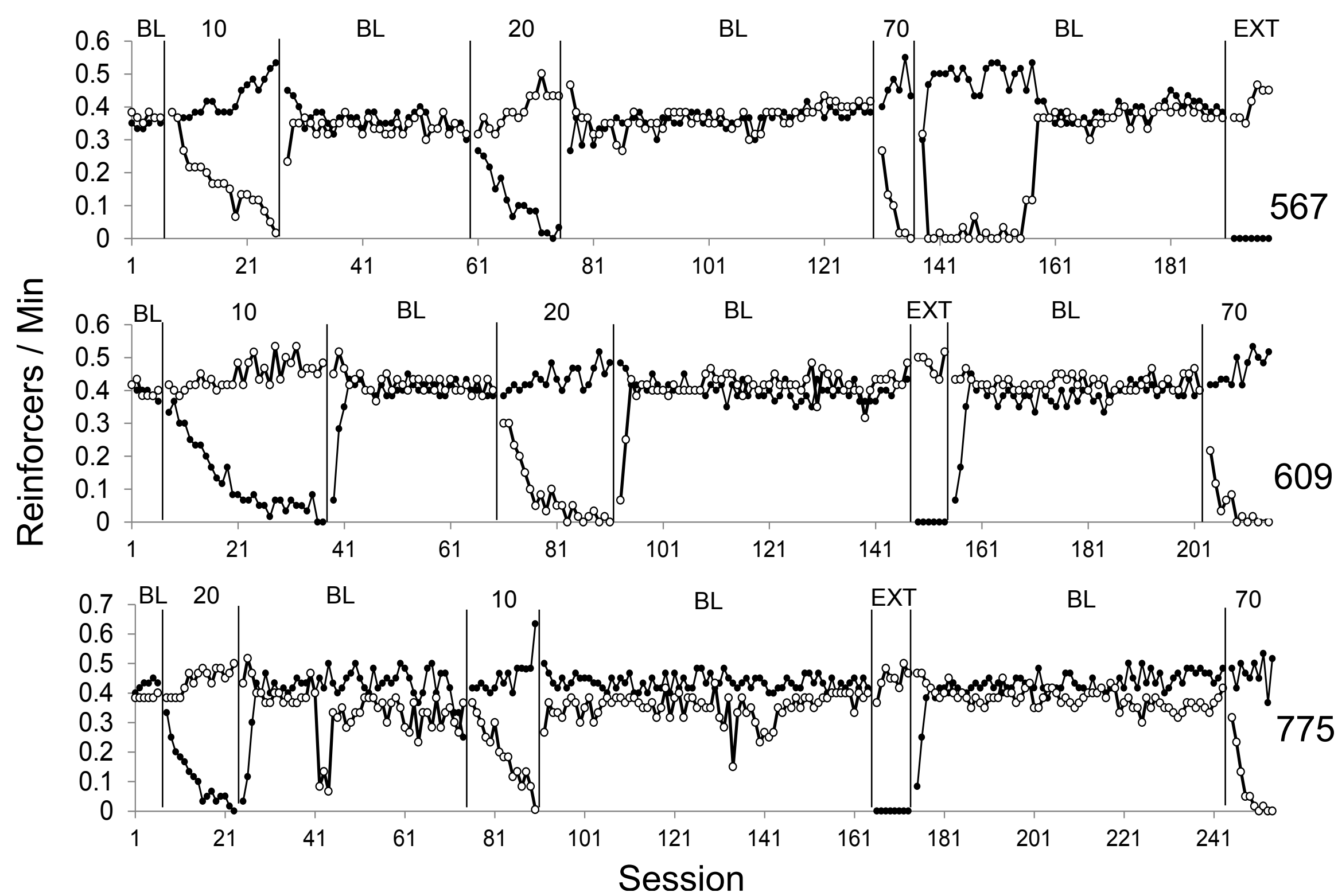

Figure 3. Reinforcement rates for each of the component schedules for the last 6 session s of the first baseline and subsequent experimental conditions. Filled data points represent the schedule correlated with green, open data points represent the schedule correlated with red. BL and EXT represent baseline and extinction, respectively. 10, 20, and 70 represent $10 \%, 20 \%$, and $70 \%$ thinning conditions, respectively. 

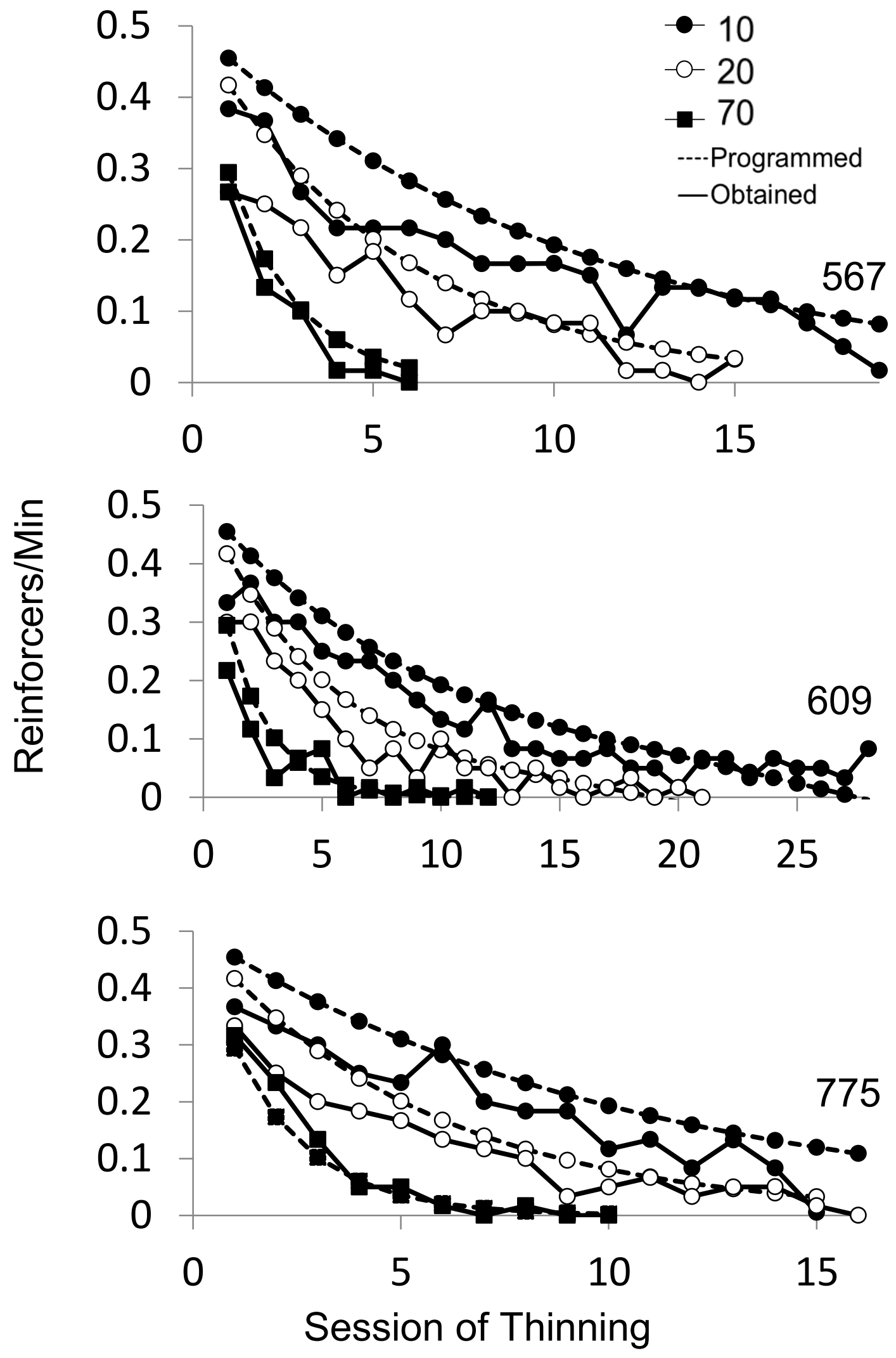

Figure 4. Programmed and obtained reinforcement rates for all thinning conditions. 

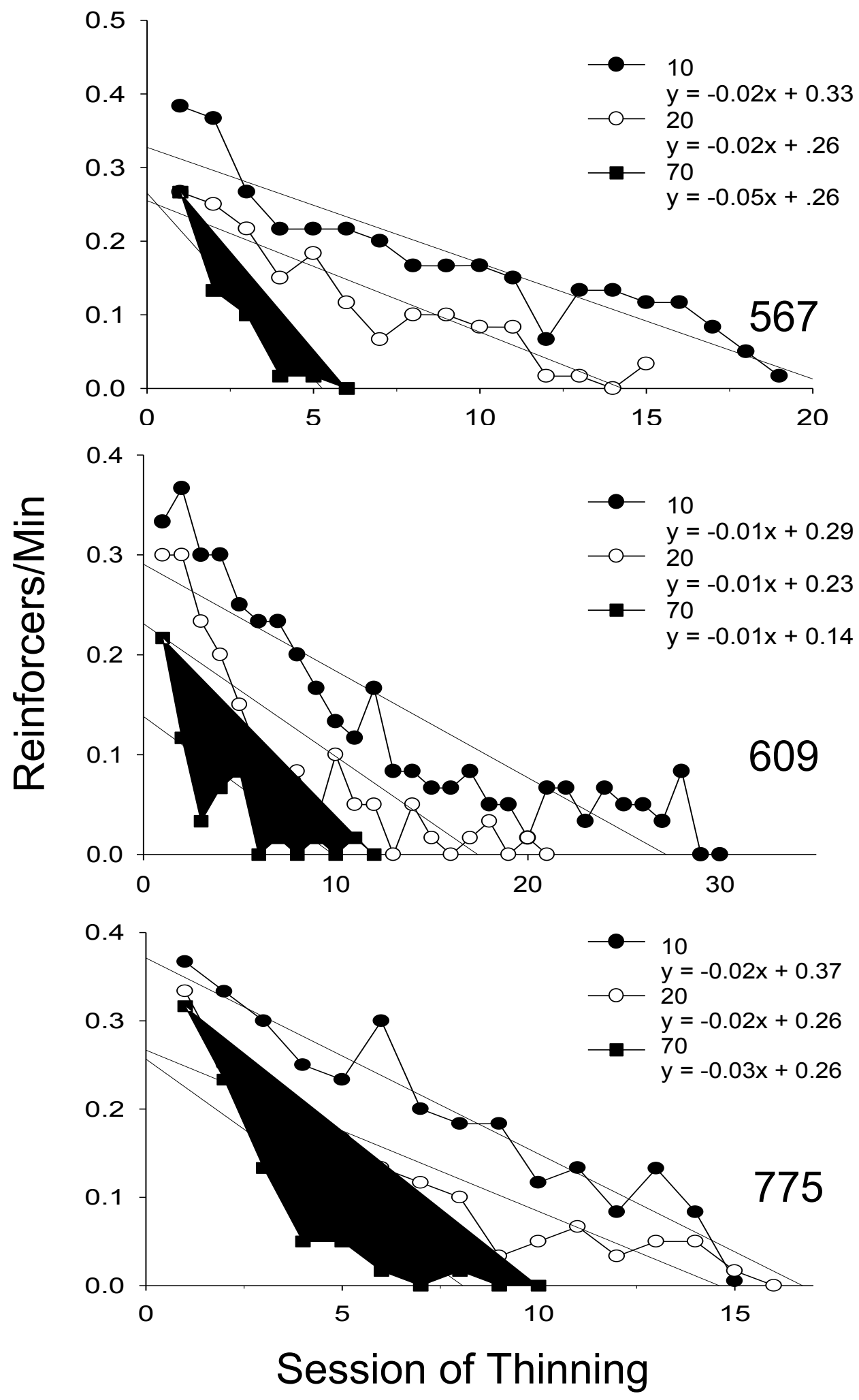

Figure 5. Obtained reinforcement rates for all thinning conditions with linear trend lines. 


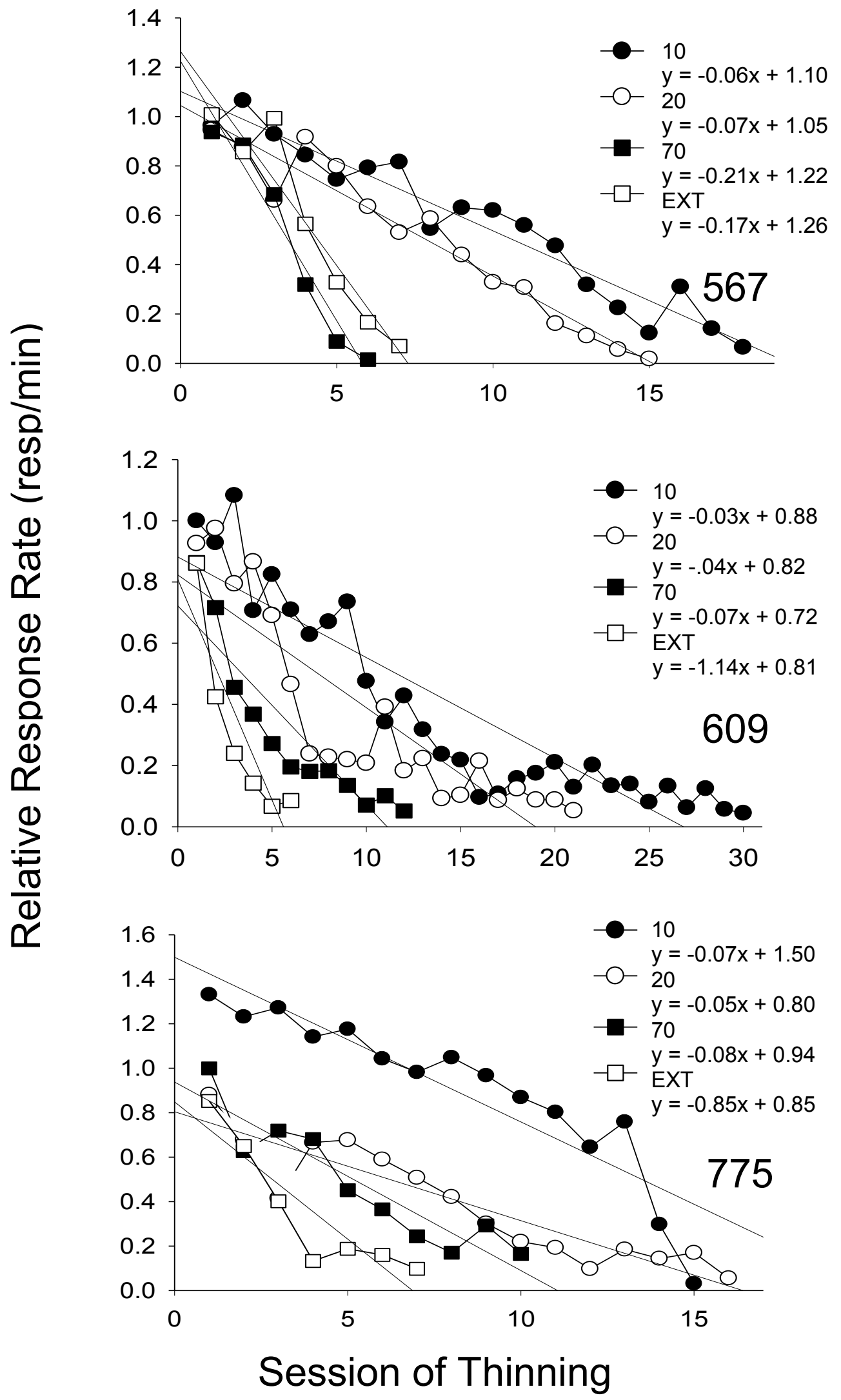

Figure 6. Response rates relative to the mean of the last 6 sessions of the preceding baseline for extinction and all thinning conditions. 
$10 \%$
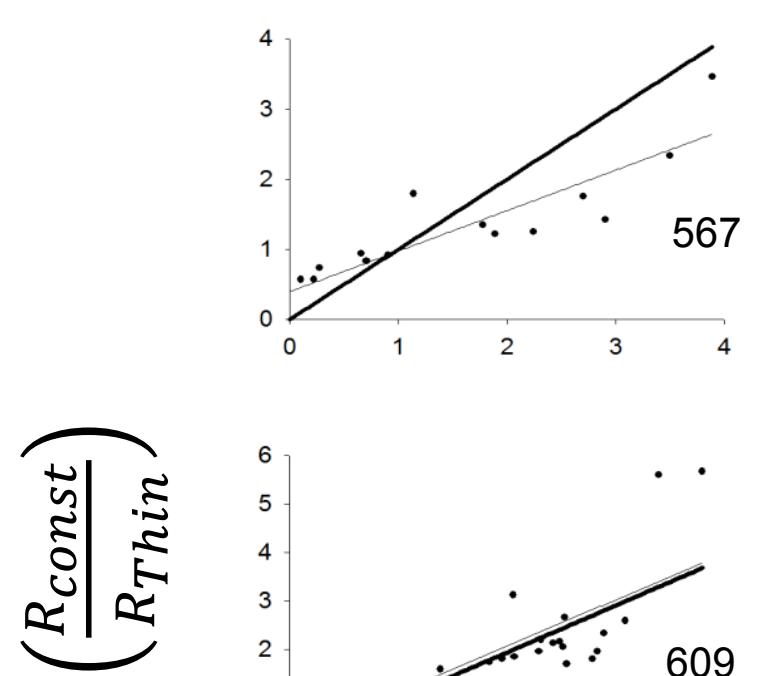

b

이

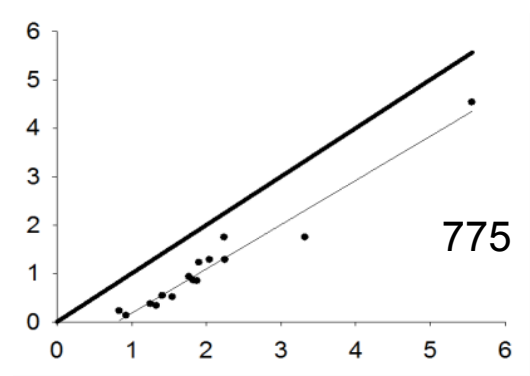

$20 \%$
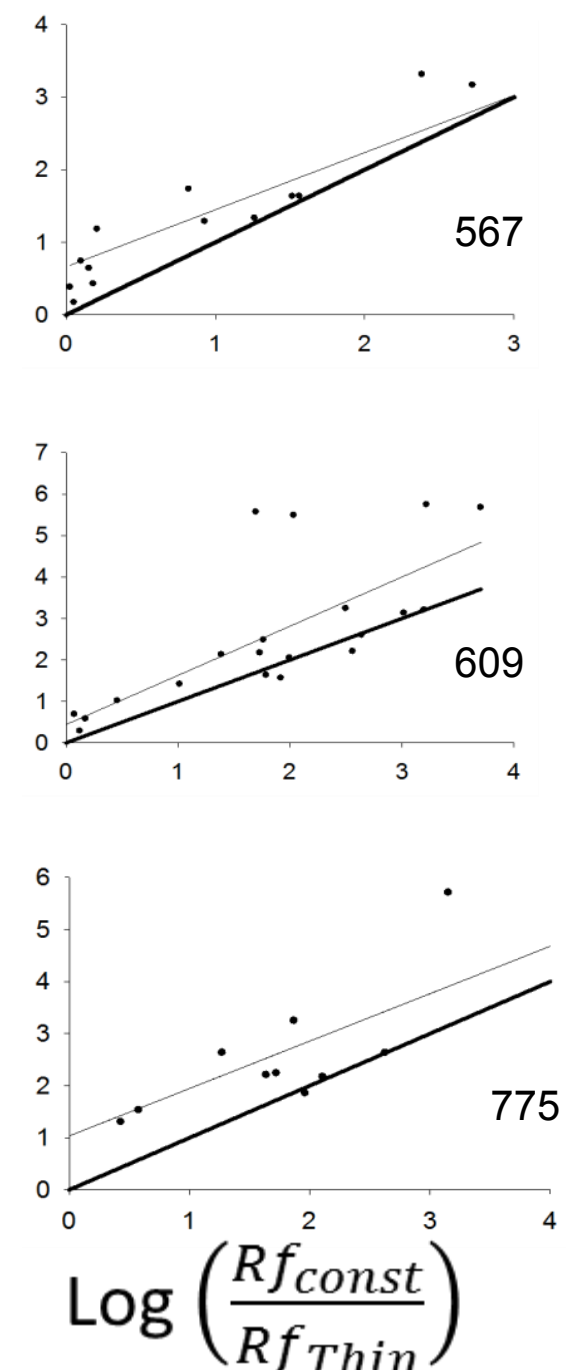

$70 \%$
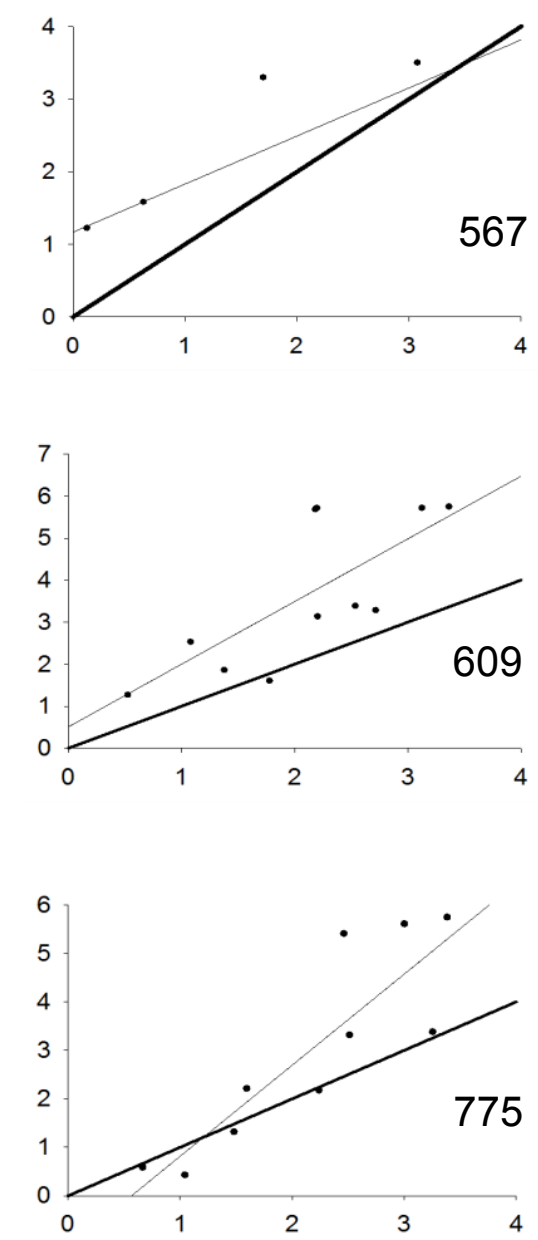

Figure 7. Log response ratios as a function of log reinforcement ratios. The thin line represents the obtained matching function. The bold line identifies strict matching. "Const" identifies the constant schedule, "Thin" identifies the thinned schedule. 